\title{
Quantum information processing
}

Two become one

\section{Neergaard-Nielsen, Jonas Schou}

\section{Published in:}

Nature Photonics

Link to article, DOI:

10.1038/nphoton.2013.169

Publication date:

2013

Link back to DTU Orbit

Citation (APA):

Neergaard-Nielsen, J. S. (2013). Quantum information processing: Two become one. Nature Photonics, 7(7), 512-513. https://doi.org/10.1038/nphoton.2013.169

\section{General rights}

Copyright and moral rights for the publications made accessible in the public portal are retained by the authors and/or other copyright owners and it is a condition of accessing publications that users recognise and abide by the legal requirements associated with these rights.

- Users may download and print one copy of any publication from the public portal for the purpose of private study or research.

- You may not further distribute the material or use it for any profit-making activity or commercial gain

- You may freely distribute the URL identifying the publication in the public portal

If you believe that this document breaches copyright please contact us providing details, and we will remove access to the work immediately and investigate your claim 


\section{Quantum information processing}

\section{Two become one}

Scientists experimentally demonstrate a scheme for quantum joining, which allow the number of qubits encoded per photon to be varied while keeping the overall quantum information constant. The inverse 'splitting' process is also proposed.

\section{Jonas Schou Neergaard-Nielsen}

Reporting in Nature Photonics, Chiara Vitelli and colleagues present a way of transferring quantum information contained in two photons into a single photon, and vice versa ${ }^{1}$. The two processes can be seen as a kind of multiplexing and demultiplexing, as shown in Fig. 1, that could potentially improve the quantum communication rate across lossy channels by limiting the number of fragile photons being transmitted.

Quantum bits, or qubits, are the quantum analogues of the bits of standard information technology in the sense that they are both the fundamental logical units of information of their respective domains. As is the case for bits, a logical qubit can also be encoded into a variety of physical objects and properties, such as energy levels of trapped atoms and solid-state artificial atoms, flux, energy or charge of superconducting circuits, spins of electrons and atomic nuclei, and so on. These are all examples of stationary qubits, useful for storage and processing of quantum information. For communication purposes, for example, in a quantum network, light is obviously much more suitable, and single photons are the obvious carriers of flying qubits (although other quantum states of light, like coherent states, are also good candidates ${ }^{2}$.

A photon is a single excitation of an electromagnetic field mode, and as such it has a wealth of degrees of freedom, such as the spatio-temporal mode, wavelength, polarization and orbital angular momentum, all of which in principle can be used for the encoding of a qubit. In experiments to date, polarization has been preferred due to its ease of manipulation with waveplates and polarizing beam splitters. The two orthogonal polarizations are perfect for the encoding of a qubit with values 0 or 1 , represented as the horizontal $(\mathrm{H})$ and vertical $(\mathrm{V})$ polarizations, respectively.

While single photons can be utilized for quantum cryptography, multiple entangled photons are required to fully unlock the power of quantum information processing. Entanglement is the fundamental nonlocal correlation that gives rise to quantum parallelism and allows a quantum computer to perform certain calculations exponentially faster than classical computers. Since the first realization of polarization-entangled pairs of photons in $1995^{3}$, researchers have worked tirelessly to create entangled states of ever more photons through the process of spontaneous parametric down-conversion. Due to the process' probabilistic nature, this quickly gets very slow and experimentally demanding. Furthermore, if a multi-photon state is transmitted over a lossy channel, it takes only the loss of one of the constituent 
photons to destroy the information. For this reason, it is sensible to look for alternative methods for encoding the quantum information. That is where the other degrees of freedom come into play.

While polarization is strictly two-dimensional, other degrees of freedom are more spacious, offering additional slots - infinitely many, in principle - for the encoding of multiple qubits onto individual photons. Formally, they provide a larger Hilbert space. As an example, take the spatial degree of freedom and imagine a series of four parallel optical paths. A single photon travelling down those paths can contain the same information as two polarization-encoded photons, with each path corresponding to one of the four two-qubit combinations $\mathrm{HH}, \mathrm{HV}, \mathrm{VH}$, and $\mathrm{VV}$. The single photon then carries a four-dimensional qudit. This pattern could of course be extended to more than two qubits.

There are thus two distinct approaches for expanding the available photonic quantum code space - increase the number of photons or use higher-dimensional degrees of freedom for encoding qudits instead of qubits into each photon. These two approaches can also be combined and multiple degrees of freedom can be used simultaneously, leading to so-called hyperentanglement ${ }^{4,5}$. Many advanced experiments have been performed, demonstrating such intricate control over the encoding of quantum information in light ${ }^{6}$. However, the distribution of information between those two forms of higher-dimensional coding has always remained static.

Vitelli and colleagues now introduce a way to dynamically convert between them ${ }^{1}$. They start from two qubits encoded in the polarization state of two independent photons. Through a process they have termed quantum joining, as schematically shown in Fig. 1, they transfer this information unchanged onto a single photon. The resulting photon's 4-dimensional qudit is encoded in its polarization and two separate spatial modes. To spell it out, the information is transferred from a Hilbert space of size 2 (photons) $\times 2$ (polarizations) to a Hilbert space of size 1 (photon) $\times 2$ (polarizations) $\times 2$ (paths).

In order to do this, it is necessary for the input photons to interact, but the curse (and the blessing) of photons is that they are very reluctant to interact with each other. If one is willing to sacrifice deterministic operation of one's device, however, it can be done through measurement-induced nonlinearity as per the linear optical quantum computing (LOQC) paradigm ${ }^{7}$. There, with a combination of extra ancilla photons, beam splitters and single photon detectors, a controlled-NOT gate can be implemented between two photons, a control and a target, conditioned on the right combination of detection events (Fig. 2). The CNOT gate entangles the two photons, but by measuring and thereby erasing the control photon afterwards, its initial quantum state can be transferred to the target photon ${ }^{8}$. Vitelli et al. use this approach to transfer the polarization qubit of input photon 1 (c) onto the polarization of the output photon. Before doing that, they transformed the polarization qubit of photon 2 (t) into a path qubit by simply separating the $\mathrm{H}$ and $\mathrm{V}$ polarizations into two different paths, $t_{1}$ and $t_{2}$, on a polarizing beam splitter and rotating on a wave plate the reflected $\mathrm{V}$-polarized beam into $\mathrm{H}$ polarization. A clever trick is then the subsequent 
dual CNOT gates, where the two paths of photon 2 are the targets and photon 1 acts as the control qubit in both gates. In this way, the polarization state of photon 1 is transferred to the polarization of a single output photon, no matter which of the two paths it occupies. This completes the re-encoding $\mathrm{H}_{\mathrm{c}} \mathrm{H}_{\mathrm{t}} \rightarrow \mathrm{H}_{\mathrm{t} 1}, \mathrm{H}_{\mathrm{c}} \mathrm{V}_{\mathrm{t}} \rightarrow \mathrm{H}_{\mathrm{t} 2}, \mathrm{~V}_{\mathrm{c}} \mathrm{H}_{\mathrm{t}} \rightarrow \mathrm{V}_{\mathrm{t} 1}, \mathrm{~V}_{\mathrm{c}} \mathrm{V}_{\mathrm{t}}$ $\rightarrow \mathrm{V}_{\mathrm{t} 2}$.

Vitelli et al. succeeded in demonstrating their suggested protocol for a number of different combinations of input qubits. The experimental set-up was carefully designed to be compact and stable since interferometric stability is required for the state analysis of a path-encoded quantum state. Even then, the fidelity obtained between the measured and the desired output states was only $75 \%$ on average, a rather modest value. This low fidelity was not due to a flaw in the protocol, but rather due to limited indistinguishability of the three photons which were originally produced in two separate down-conversion processes. A major limitation of the protocol itself, however, is its probabilistic operation which it shares with related LOQC schemes; even in the optimal case, it only succeeds one out of eight times.

While the experimenters on the team had their hands full with carrying out the quantum joining protocol, the theorists went a bit further and suggested ways to extend it to multiple input photons, adding correspondingly more possible paths to the single output photon ${ }^{1}$. They also proposed a possible implementation of the opposite process, which they call quantum splitting, where the qudit state of a single photon is split into two or more polarization-encoded qubits. With these concepts at hand, they assert that their quantum joiner and splitter serve as a multiplexer and demultiplexer of quantum information. This assertion makes sense in the context of quantum networks with high channel losses where it is advantageous to transmit information in as few fragile photons as possible. For sufficiently high channel losses, this advantage could overcome the disadvantage of limited success probability of the multiplexing and demultiplexing stages.

On the other hand, in terms of physical channels (spatio-temporal modes), the joined state requires the same number of channels as the input state or more - at least in the current implementation with path-encoding. It would be interesting to see a more genuine quantum multiplexing realization where a single photon carries a high-dimensional qudit on a single spatial mode. This could for example be done by taking advantage of the orbital angular momentum (OAM) of a light beam, which in principle offers an infinite code space. Multiplexing in OAM was recently demonstrated for terabit classical data transmission ${ }^{9}$, and the groups behind the current result have themselves worked extensively with quantum states of OAM modes, among other things preparing a four-dimensional polarization-OAM qudit state of a single photon ${ }^{10}$. The prospects of extending the quantum state joining and splitting protocols to the OAM degree of freedom therefore seem good, and while there would still be the limitation of low success rate along with other practical problems, it could eventually provide a platform for high-capacity multiplexed quantum state transmission over lossy channels. 
Jonas Schou Neergaard-Nielsen is at the Department of Physics, Technical University of Denmark, Fysikvej, 2800 Kgs. Lyngby, Denmark. e-mail: jsne@fysik.dtu.dk

\section{References}

1. Vitelli, C. et al. Nature Photon. 7, 521-526 (2013).

2. Jeong, H. \& T. C. Ralph in Quantum Information with Continuous Variables of Atoms and Light (Imperial College Press, 2007).

3. Kwiat, P. G. et al. Phys. Rev. Lett. 75, 4337-4341 (1995).

4. Barreiro, J. T., Langford, N. K., Peters, N. A. \& Kwiat, P. G. Phys. Rev. Lett. 95, 260501 (2005).

5. Gao, W.-B. et al. Nature Phys. 6, 331-335 (2010).

6. Pan, J.-W. et al. Rev. Mod. Phys. 84, 777-838 (2012).

7. Knill, E., Laflamme, R. \& Milburn, G. J. Nature 409, 46-52 (2001).

8. Pittman, T. B., Jacobs, B. C. \& Franson, J. D. Phys. Rev. A 64, 062311 (2001).

9. Wang, J. et al. Nature Photon. 6, 488-496 (2012).

10. Nagali, E., Sansoni, L., Marrucci, L., Santamato, E. \& Sciarrino, F. Phys. Rev. A 81, 052317 (2010).

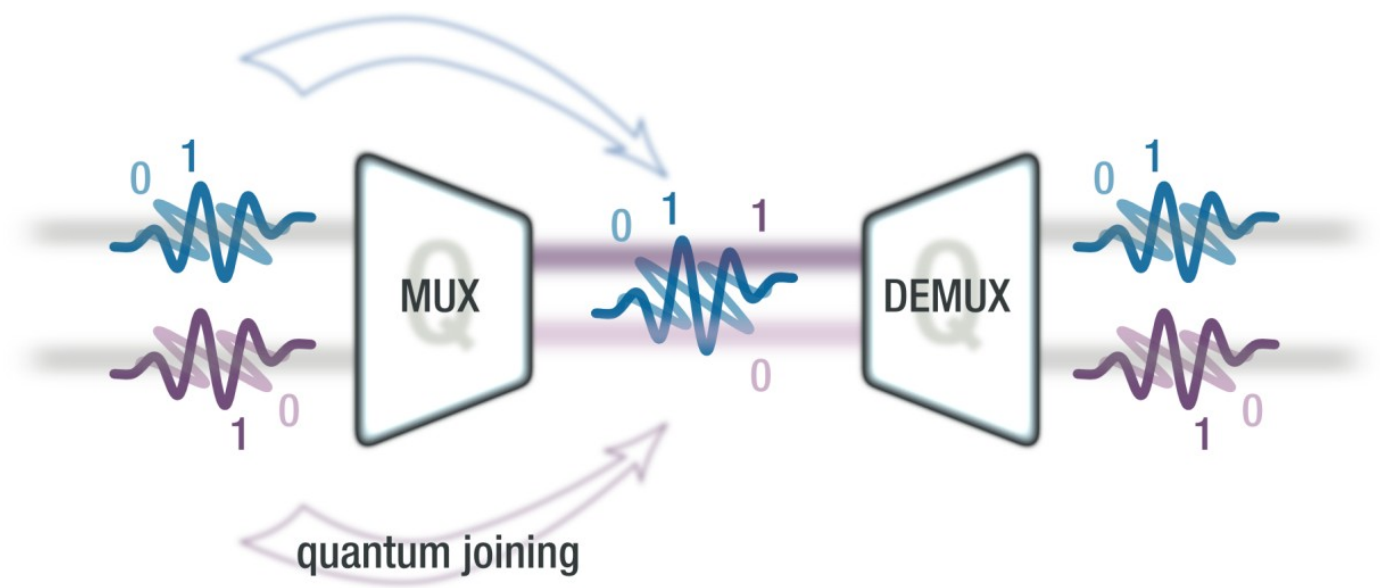

Figure 1 The quantum joining process transfers the qubits carried by two incoming photons to a four-dimensional qudit of a single photon. This "quantum multiplexer" can be combined with the inverse process for establishing a more loss-tolerant data transmission. 


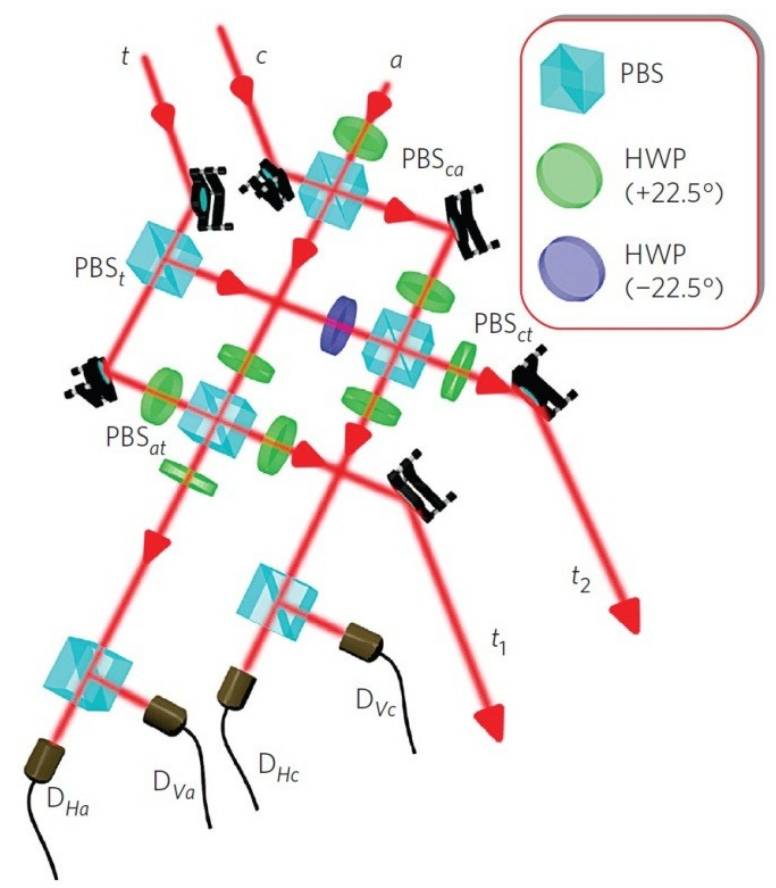

Figure 2 Conceptual scheme of an implementation of the quantum joining of two polarization qubits (t and $c$ ) onto a path-polarization qudit $\left(t_{1}-t_{2}\right)$. The scheme employs an ancilla photon, polarizing beam splitters, waveplates and photon detectors. 